\title{
Supporting Information for \\ Does Dipolar Motion of Organic Cations Affect Polaron Dynamics and Bimolecular Recombination in Halide Perovskites?
}

Kyle T. Munson, John R. Swartzfager, Jianing Gan, and John B. Asbury*

Department of Chemistry, The Pennsylvania State University, University Park, PA 16802, USA.

*Corresponding Author: jba11@psu.edu

\section{Table of Contents:}

1. Experimental Procedures

2. Scanning Electron Microscopy

3. TRIR Measurements

4. References

\section{Experimental Procedures}

\section{Sample Preparation}

For TRIR measurements, $\mathrm{BaF}_{2} /$ mesoporous alumina substrates were prepared by spincoating a 4 wt \% solution of $\mathrm{Al}_{2} \mathrm{O}_{3}$ nanoparticles in isopropyl alcohol (6000 rpm, $30 \mathrm{sec}$ ) unto oxygen plasma cleaned $\mathrm{BaF}_{2}$. The resulting $\mathrm{Al}_{2} \mathrm{O}_{3} / \mathrm{BaF}_{2}$ films were then annealed at $450{ }^{\circ} \mathrm{C}$ for 1 hr. After annealing, the substrates were treated with an oxygen plasma for 4 min to ensure complete removal of organic materials.

Perovskite films were deposited using a one-step deposition method in which a 0.45 M DMSO solution containing $\mathrm{PbBr}_{2}$ and $\mathrm{MABr}, \mathrm{FABr}$, or $\mathrm{CsBr}$ was deposited onto the $\mathrm{Al}_{2} \mathrm{O}_{3} / \mathrm{BaF}_{2}$ substrates by spin-coating at 3000 rpm for 1 min. For $\mathrm{CsPbBr}_{3}$ perovskites, $\mathrm{CsBr}$ was dissolved in DMSO first by constantly stirring at $40{ }^{\circ} \mathrm{C}$ before the addition of $\mathrm{PbBr}_{2}$. As-cast perovskite films were annealed for $10 \mathrm{~min}$ at $110^{\circ} \mathrm{C}$. The perovskite films were then treated with a $5 \mathrm{mM}$ solution of TPPO dissolved in chlorobenzene to remove surface defects.

\section{Visible Absorption Measurements}

UV-Vis absorption spectra were collected with a Beckman DU 520 UV/Vis spectrometer. The spectra were background subtracted by the $\mathrm{BaF}_{2} / \mathrm{Al}_{2} \mathrm{O}_{3}$ substrate absorption. 


\section{FTIR Measurements}

FTIR absorption spectra were acquired with a Digilab Varian FTS 7000 Series FTIR spectrometer equipped with a liquid $\mathrm{N}_{2}$ cooled MCT detector. All spectra were the average of 1000 scans between the spectral range of $1300-2000 \mathrm{~cm}^{-1}$. The spectra were background subtracted by the $\mathrm{CaF}_{2}$ substrate absorption and baseline corrected using a third order polynomial.

\section{Nanosecond-TRIR Measurements}

Nanosecond-TRIR spectroscopy experiments were performed using a mid-IR transient absorption spectrometer (insplRe) from Magnitude Instruments (State College, PA). The instrument consisted of a nanosecond Nd:YAG laser with second harmonic generation (532 nm), which was used to excite the perovskite films. The pulse energy density used for all ns-TRIR experiments was $500 \mathrm{~nJ} / \mathrm{cm}^{2}$ per pulse. The infrared probe light was generated with a $\mathrm{MoSi}_{2}$ infrared element. The resulting continuous-wave infrared probe radiation was focused on the sample, overlapped with the laser pulse, and then dispersed into a monochromator. The transient absorption signal was collected with a liquid $\mathrm{N}_{2}$ cooled mercury cadmium telluride (MCT) photovoltaic detector.

\section{Ultrafast-TRIR Measurements}

Ultrafst-TRIR measurements were collected using an ultrafast laser setup previously described. ${ }^{1}$ In brief, 100 fs, $800 \mathrm{~nm}$ pulses were produced by an amplified Ti:Sapphire laser (Vitesse, Coherent; Santa Clara, CA/Integra-C, Quantronix;Santa Clara CA). These pulses were used to pump two separate optical parametric amplifiers (OPAs) (TOPAS, Light Conversion Ltd; Vilnius, Lithuania). The first OPA was tuned to $532 \mathrm{~nm}$ while the other was tuned to produce midIR light from 1500-1600 $\mathrm{cm}^{-1}$. Samples were pumped using the $532 \mathrm{~nm}$ pulse while the mid-IR pulse was used as a probe source. Mid-IR probe pulses were dispersed though a monochromator (Triax, Horiba; Kyoto, Japan) and detected using a 32X2 element MCT array detector (Infrared Associates/ Infrared Systems Development; Stuart, Florida).

\section{Scanning Electron Microscopy}

Scanning electron micrographs were used to characterize film thicknesses to quantify the charge recombination kinetics of large polarons using the bimolecular recombination model. 
Scanning electron micrographs were acquired with a ThermoFisher Scientific Verios scanning electron microscope with a landing energy of $1.00 \mathrm{keV}$. Figure $\mathbf{S} 1$ shows a cross-sectional SEM images of an iridium coated ( $\sim 15 \AA$ thick) perovskite films. From the cross-sectional images, we estimated the thickness of the perovskite films examined in this work to be $400 \pm 10 \mathrm{~nm}, 430 \pm$ 20nm, and $450 \pm 10 \mathrm{~nm}$ for $\mathrm{CsPbBr}_{3}, \mathrm{MAPbBr}_{3}$, and $\mathrm{FAPbBr}_{3}$ films, respectively.
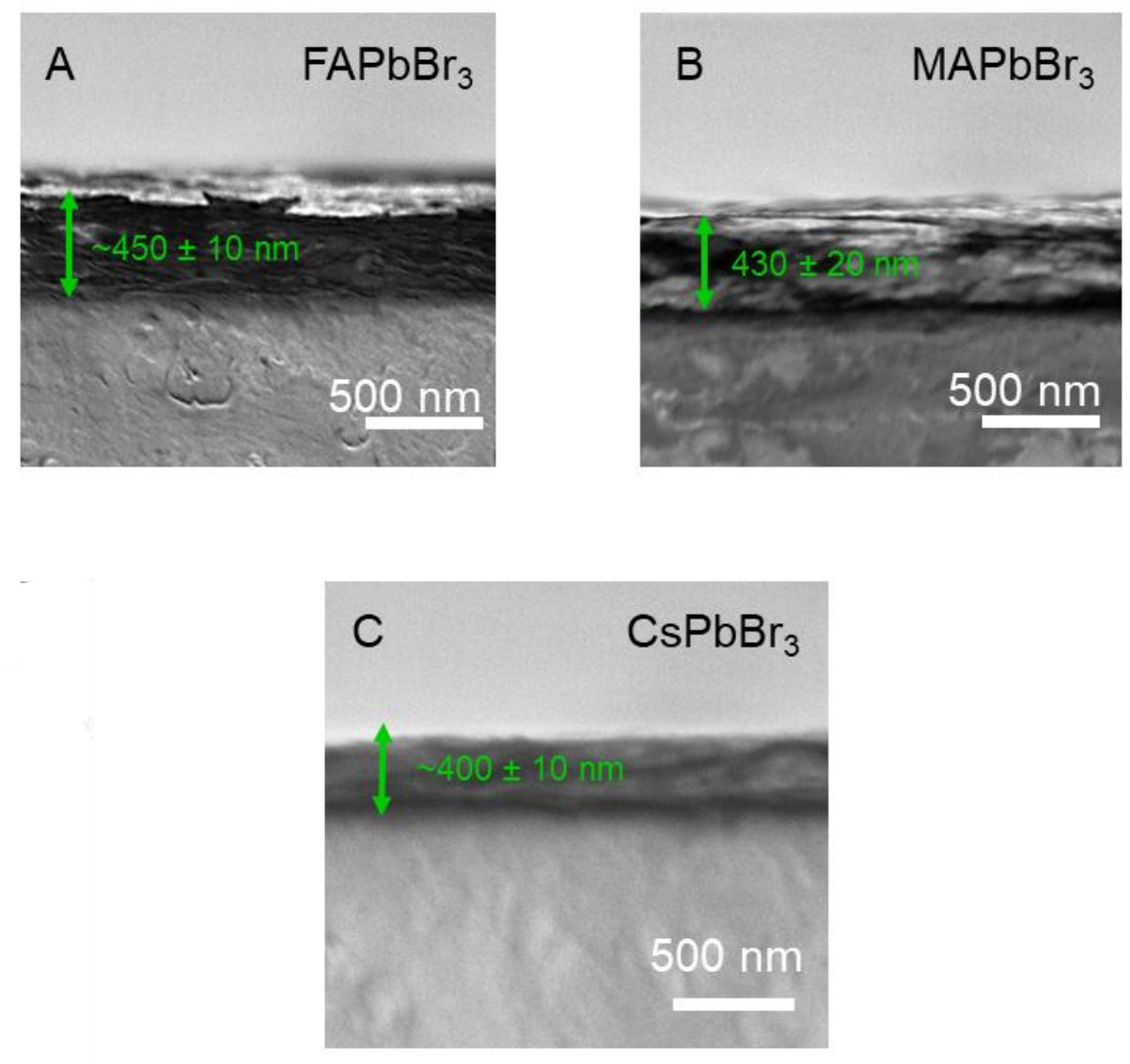

Figure S1. Cross-sectional SEM images of (A) $\mathrm{FAPbBr}_{3}$, (B) $\mathrm{MAPbBr}_{3}$, and (C) $\mathrm{CsPbBr} 3$ perovskite films. The scale bars are $500 \mathrm{~nm}$.

\section{TRIR Measurements}

Figure S2 shows expanded regions of the TRIR spectra presented in Figure 2A, which highlight the vibrational features of MA and FA cations within the perovskite films. We note that similar excited-state vibrational features also were observed in the TRIR spectra of $\mathrm{MAPbl}_{3}$ and $\mathrm{FAPbl}_{3}$ films. ${ }^{2}$ These features were thought to arise from the coupling of the vibrational and electronic coordinates of the material, similar to infrared activated vibrational (IRAV) modes observed in conjugated polymers. 

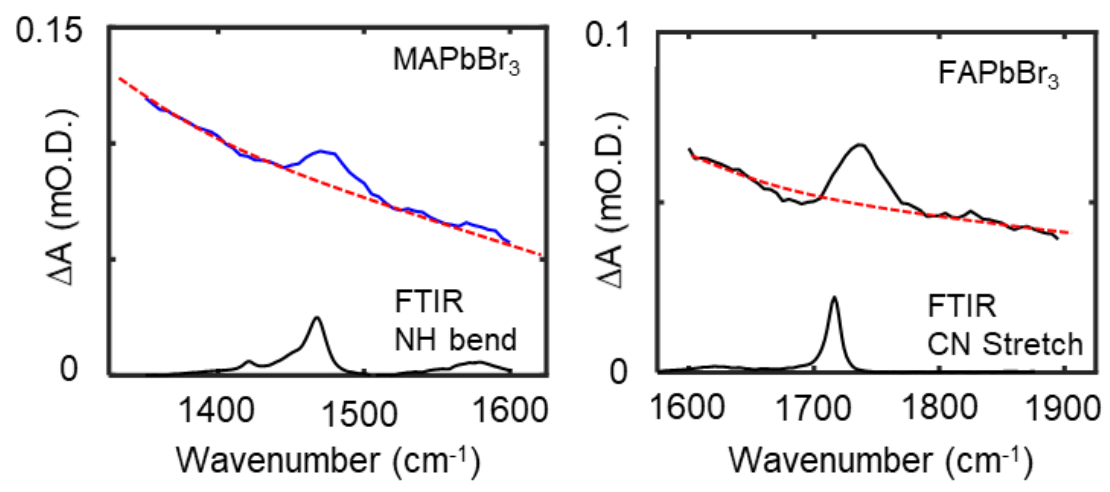

Figure S2. Expanded regions of the TRIR spectra presented in Figure 2A highlighting the N$\mathrm{H}$ bend (left) and $\mathrm{CN}$ stretch (right) vibrational modes of MA and FA cations, respectively.

Figure S3 compares the TRIR spectra of a representative perovskite film collected at several time delays. The spectra have been normalized to facilitate comparison of their frequency dependent shapes. The TRIR spectra of the film undergo negligible change in shape with increasing time delay, demonstrating that photo-generated charges remain in delocalized large polaronic states throughout their lifetime.

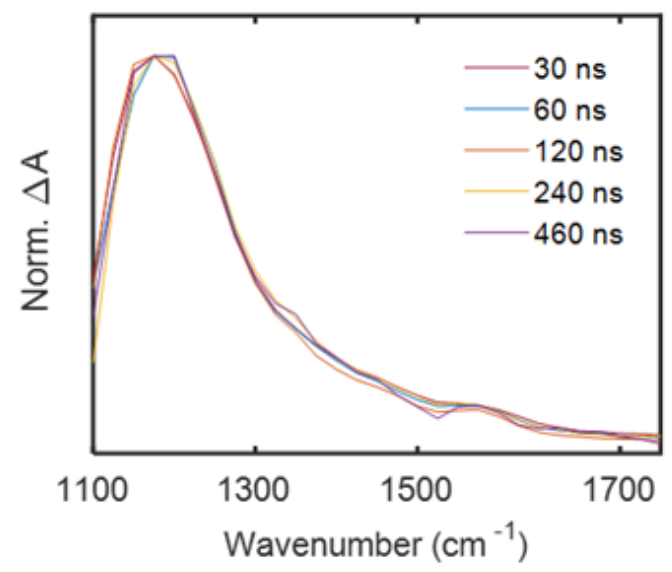

Figure S3. TRIR spectra of a representative $\mathrm{CsPbBr}_{3}$ perovskite film collected at several time delays. The spectra have been normalized to facilitate a comparison of their frequency dependent shapes. The spectra undergo negligible change in shape with increasing time delay, indicating that photo-generated charges remain in large polaronic states throughout their lifetime. 
The TRIR spectra of $\mathrm{MAPbBr}_{3}, \mathrm{FAPbBr}_{3}$, and $\mathrm{CsPbBr}_{3}$ films were fit using Equations 1 and 2 of the main text to evaluate their spectral linewidth above the absorption maximum. The best-fit parameters for the fits appear in Table S1.

Table S1. List of best fit parameters for the spectral fits in Figure $2 \mathrm{C}$ in the main text.

\begin{tabular}{|c|c|c|c|}
\hline Sample & Delocalization Length (R) & Rel. Delocalization Length & $3 \times \mathrm{E}_{\mathrm{p}}(\mathrm{eV})$ \\
\hline $\mathrm{MAPbBr}_{3}$ & $9.5 \pm .1$ & $1.0+/-0.1$ & $.14 \pm .1$ \\
\hline $\mathrm{FAPbBr}_{3}$ & $9.7 \pm .2$ & $1.0+/-0.2$ & $.14 \pm .1$ \\
\hline $\mathrm{CsPbBr}_{3}$ & $9.6 \pm .3$ & $1.0+/-0.3$ & $.14 \pm .1$ \\
\hline
\end{tabular}

To determine the molar extinction coefficient $(\alpha)$ of the mid-IR polaron absorption signature, we measured $\triangle \mathrm{A}$ as a function of initial polaron concentration $[\mathrm{P}]_{0}$. Power-dependent TRIR measurements were used to generate different initial polaron concentrations. Representative power-dependent polaron decay kinetics are shown in Figure S4A. The initial polaron concentration was estimated from the maximum intensity of the decay kinetics measured near the instrument response ( $\sim \mathrm{ns}$ ). This estimation is valid at sufficiently low excitation densities at which the relationship between $\Delta \mathrm{A}_{0}$ and the absorbed photon density, Eph, is linear (see Figure S4B). Therefore, $\alpha$ was determined from the slope of a linear fit to $\Delta \mathrm{A}_{0}$ vs Eph. Table $\mathbf{S} 2$ lists the extinction coefficients obtained for each perovskite sample examined in this work and their associated errors. 

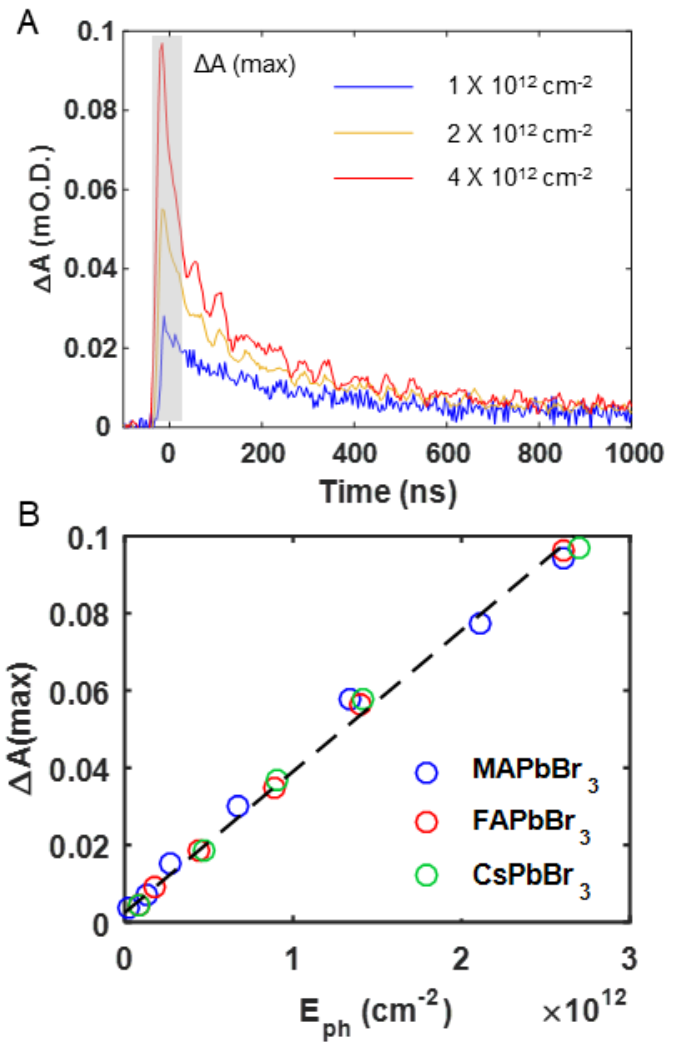

Figure S4. (A) representative time-resolved mid-IR decays of a $\mathrm{FAPbBr}_{3}$ film measured at different pump fluences. The decays were obtained by integrating the frequency range between $1000-1800 \mathrm{~cm}^{-1}$. The shaded box indicates the region near the IRF where $\triangle \mathrm{Amax}$ was determined. (B) Power dependence of $\Delta A \max$ as a function of absorbed photon density, Eph. $\mathrm{A}$ linear dependence of $\triangle \mathrm{A}$ on Eph is observed (dotted black line).

Table S2. Relative extinction coefficients. List of the fitting parameters obtained from the linear fits in Figure S4B

\begin{tabular}{|c|c|}
\hline Sample & $\alpha\left(\right.$ O.D. cm $\left.{ }^{2}\right)$ \\
\hline $\mathrm{MAPbBr}_{3}$ & $(3.5 \pm .2) \times 10^{-17}$ \\
\hline $\mathrm{FAPbBr}_{3}$ & $(3.7 \pm .2) \times 10^{-17}$ \\
\hline $\mathrm{CsPbBr}_{3}$ & $(3.6 \pm .2) \times 10^{-17}$ \\
\hline
\end{tabular}


To determine if higher-order annihilation processes contributed to our ultrafast TRIR measurements, we measured the decay of large polarons on the sub-ns timescale following optical excitation at $532 \mathrm{~nm}\left(2 \times 10^{17} \mathrm{~cm}^{-3}\right)$. Figure S5 displays ultrafast-TRIR decay kinetics of $\mathrm{CsPbBr}_{3}, \mathrm{MAPbBr}_{3}$, and $\mathrm{FAPbBr}_{3}$ thin films obtained by integrating the spectral region between 1500 and $1600 \mathrm{~cm}^{-1}$. The comparison demonstrates that the TRIR signal does not decay significantly within the first 100 ps after photoexcitation, indicating that higher-order annihilation processes do not contribute to the ultrafast measurements presented in Figure 4 of the main text.

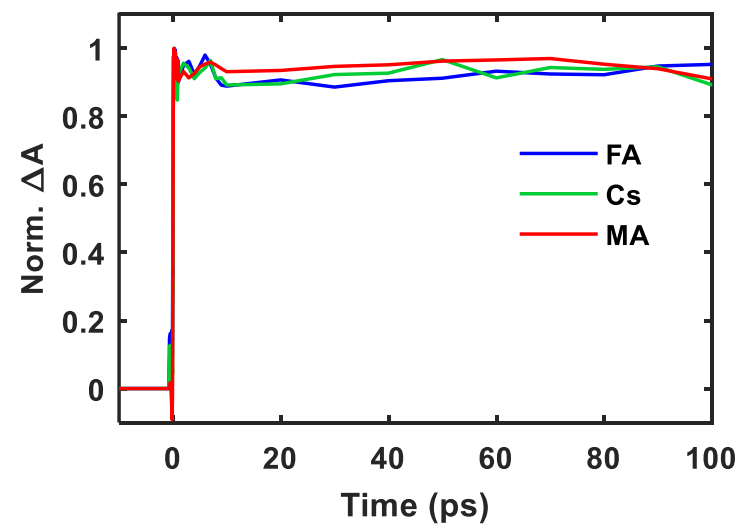

Figure S5. Ultrafast-TRIR decay traces of $\mathrm{CsPbBr}_{3}$, $\mathrm{MAPbBr} 3$, and $\mathrm{FAPbBr}_{3}$ thin films obtained by integrating the spectral region between 1500 and $1600 \mathrm{~cm}^{-1}$ following photoexcitation at $532 \mathrm{~nm}\left(2 \times 10^{17} \mathrm{~cm}^{-3}\right)$.

To illustrate that carrier cooling does not contribute significantly to our ultrafast-TRIR measurements, we measured the formation of large polarons in a $\mathrm{MAPbBr}_{3}$ film as a function of absorbed photon density following optical excitation at $532 \mathrm{~nm}$ (Figure S6). The comparison demonstrates that the formation of large polarons does not depend on the density of absorbed photons used to excite the film. Because carrier cooling rates in halide perovskites have been shown to depend on excitation density, ${ }^{3}$ we conclude that carrier cooling does not contribute significantly to our measurements due to the absence of such a dependence in Figure $\mathbf{S 6 .}$ 


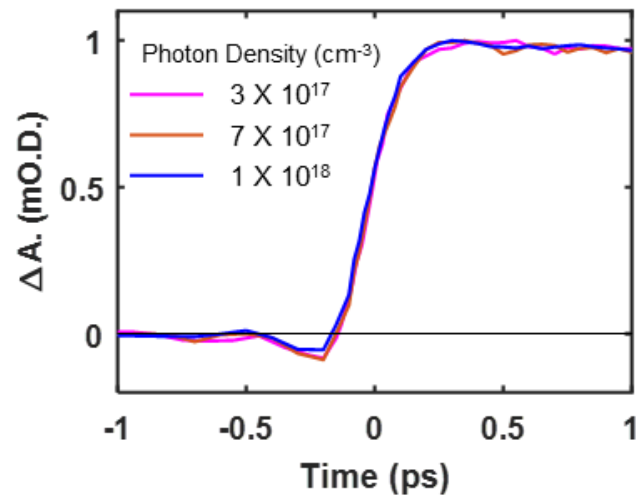

Figure S6. Power-dependent ultrafast-TRIR decay traces of a $\mathrm{FAPbBr}_{3}$ thin film obtained by integrating the spectral region between 1500 and $1600 \mathrm{~cm}^{-1}$ following photoexcitation at $532 \mathrm{~nm}$.

\section{References}

1. Barbour, L. W.; Hegadorn, M.; Asbury, J. B., Watching Electrons Move in Real Time: Ultrafast Infrared Spectroscopy of a Polymer Blend Photovoltaic Material. JACS. 2007, 129, 15884-15894.

2. Munson, K. T.; Kennehan, E. R.; Asbury, J. B., Structural origins of the electronic properties of materials via time-resolved infrared spectroscopy. J. Mater. Chem. C. 2019, 7, 58895909

3. Hopper, T. R.; Gorodetsky, A.; Frost, J. M.; Müller, C.; Lovrincic, R.; Bakulin, A. A., Ultrafast Intraband Spectroscopy of Hot-Carrier Cooling in Lead-Halide Perovskites. ACS Energy Lett. 2018, 3, 2199-2205. 